\title{
New US centre to boost comparative health research
}

Published at www.cmaj.ca on May 27

$\mathrm{H}$ ealth care experts and patient advocates in the United States say a new centre for comparative effectiveness research will advance both the efficiency and personalization of health care across the country.

Now in the early stages of setting up a board of governors, the Patient-Centered Outcomes Research Institute will receive more than US\$200 million a year starting in 2013 to research which treatments, tests and protocols for treatment, care management and delivery work best for whom and under what circumstances.

"We've had 60 years of experience with randomized control trials in an industry setting, but those types of studies operate in a regulatory framework where the focus is deciding or defining whether something can work, rather than identifying how it works in a real-world environment," says Dr. Les Paul, vice-president of clinical and scientific affairs for the National Pharmaceutical Council.

According to a 2009 report by the US Institute of Medicine, more than half of the treatments delivered in the US lack clear evidence of effectiveness. That means the evidence necessary to inform the medical decisions of consumers, patients, doctors and policy-makers is often "incomplete or unavailable."

Passed in the US Senate in late 2009 and in the House of Representatives in April, the Patient-Centered Outcomes Research Act mandated the creation of the new independent, not-for-profit institute to advance "the quality and relevance of evidence concerning the manner in which diseases, disorders, and other health conditions can ... be prevented, diagnosed, treated, monitored, and managed."

The institute will set national priorities for comparative effectiveness research and establish a research project agenda for the examination of health care products, procedures and

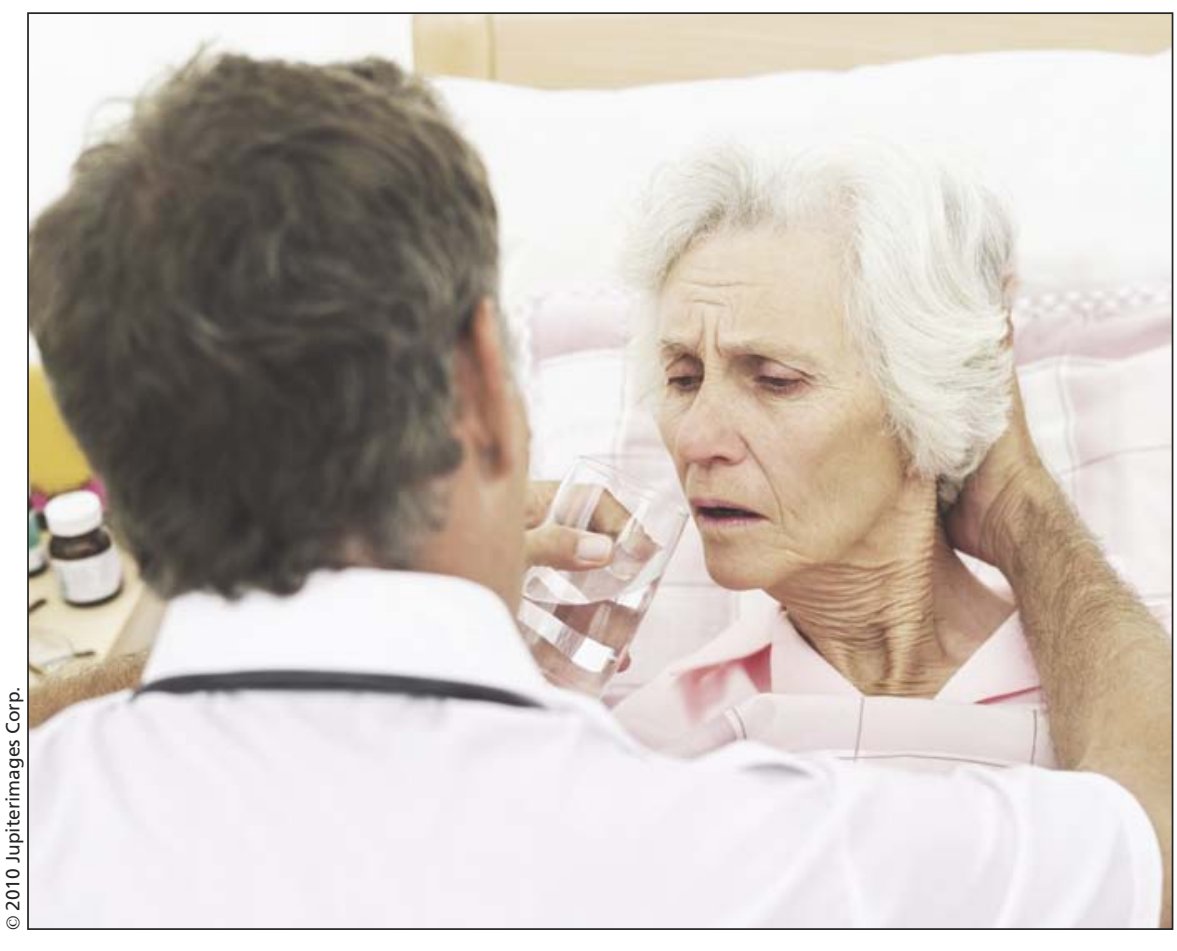

Contrary to the fears of some US health reform critics, the Patient-Centered Outcomes Research Act will not place a lower value on extending the life of an elderly patient than on extending the life of a young patient.

delivery systems. It will conduct primary research as well as systematic reviews of existing studies.

To conduct its research, the institute will contract federal agencies such as the National Institutes of Health and the Agency for Healthcare Research and Quality, as well as appropriate privatesector entities.

"The Agency for Healthcare Research and Quality has had primary responsibility for CER [comparative effectiveness research] until now, but most of that research was based on taking a single product and, without comparing it to any other products, deciding how it could best be used," says Myrl Weinberg, president of the National Health Council, which represents more than 100 national healthrelated organizations.

Rather than examining health care delivery systems or conducting product -to-product comparisons, Weinberg says the comparative research conducted by the Agency for Healthcare Research and Quality was too smallscale to effect real-world changes.

"They were looking at things like what time of day a product should be taken," she says. "Until now there wasn't the money to conduct CER on the kind of scale this new institute will be able to."

Funding for the institute will come from a Patient-Centered Outcomes Research Trust Fund, financed by transfers from two other federal medical trusts funds, starting in 2013.

"This is a huge step toward personalized medicine, where the focus shifts away from what works for most to what works best," says Weinberg.

The shift in focus will also be felt in the pharmaceutical industry, says Paul.

"We're not going to take our eyes off the ball of doing randomized control trials, but having CER being conducted 
in this way is going to create a need to refocus and reframe our research to include clinically meaningful comparators in our studies, or add things like patient-reported outcomes so we can make our research more applicable for real-world decision-makers."

Those decision-makers will be represented on the institute's 21-member board of governors, to be appointed by September by the US Government Accountability Office, the audit, evaluation and investigative arm of Congress.

The board will include the secretary of Health and Human Services, the director of the Agency for Healthcare Research Quality, and the director of the National Institutes of Health, or their designees, as well as 18 other members representing patients and health care consumers, physicians (including surgeons), agencies that run public health programs, private payers (including one health insurance plan and one self-ensuring employer), independent health services researchers and drug and device manufacturers.

The institute will have a 17 -member methodology committee, as well as expert advisory panels to assist in identifying research priorities and agendas.

Research conducted by the institute will be peer reviewed, and all findings made available to the medical community and general public within 90 days of completion.

"I don't think there's any other model, nationally or internationally, that could reflect all the positives of this, particularly the inclusiveness of involving patients in a meaningful way at different levels of the decision-making process," says Weinberg.

Once the board of governors is appointed, the biggest challenge will be setting methods and standards for comparative effectiveness research, says Paul.

"CER is a bit of a young science, so we haven't had much experience yet in how best to use the results of that research."

Opponents of health care reform have leveraged that inexperience to feed fears that the new institute could function as a kind of Orwellian "death panel," with comparative effectiveness studies being used to weigh the cost of new treatments against quality adjusted life years saved and set arbitrary thresholds on coverage.

Most recently, US Senator Chuck Grassley claimed the new institute would be used to determine the value of life. According to Grassley, the value of an 85-year-old person's life might be weighed against that of a 35year-old, and the results of comparative effectiveness research could be used to ration coverage accordingly.

The Patient-Centered Outcomes Research Act prohibits decision-makers from using the institute's findings "in a manner that treats extending the life of an elderly, disabled, or terminally ill individual as of lower value than extending the life of an individual who is young, nondisabled, or not terminally ill."

The act also mandates that no coverage decisions be based upon the institute's findings alone.

In most cases, increasing the body of evidence available to payers will increase the likelihood of treatments being approved for coverage, says Brian Sweet, chief pharmacy officer at WellPoint, a US health benefits company.
"When we're able to supplement randomized clinical trial data with CER, we get a very different picture than when we're working on the clinical trial data alone," he says. "Using CER in our formulary decision-making process even on a small scale in the past has led us to cover drugs we wouldn't have otherwise."

"The bottom line is, we don't make decisions based upon one study. We didn't do it when there was no CER available back in the early 1990s, and we're not going to do it when there are CER studies available in the next five to 10 years," Sweet adds. "This isn't about changing the way we do business; it's about supplementing the evidence we already have to get a better picture of the impact of treatments in the real world."

According to Paul and Weinberg, putting usefulness guidelines in place will prevent misuse of the institute's findings.

"There should be some sort of easyto-use guidance label that says this is how you should use these results and here are what the limitations will be," says Paul.

Weinberg says the National Health Council is currently working with the Department of Health and Human Services to develop a provision that would require the institute to create specific criteria for determining whether findings are appropriate for uses in decisions about coverage.

"There will have to be steps between the results of research and its actual application, because CER ... absolutely cannot be applied on a population basis." - Lauren Vogel, CMAJ

DOI:10.1503/cmaj.109-3269 